Journal of Engineering and Applied Sciences 15 (7): 1671-1675, 2020

ISSN: 1816-949X

(C) Medwell Journals, 2020

\title{
EEG Signal Processing Model for Eye Blink Detection
}

\author{
${ }^{1,2}$ Sebastián Poveda Zavala, ${ }^{1,2}$ Kelvin Ortíz Chicaiza, ${ }^{1,2}$ José Luis Murillo López, \\ ${ }^{1,2}$ Johanna Cerezo Ramírez and ${ }^{1,2}$ Sang Guun Yoo \\ ${ }^{1}$ Departamento de Informática y Ciencias de la Computación, \\ Escuela Politécnica Nacional, Quito, Ecuador \\ ${ }^{2}$ Smart Lab, Escuela Politécnica Nacional, Quito, Ecuador
}

\begin{abstract}
Electroencephalography devices such as the OpenBCI Cyton Biosensing board create a noninvasive and inexpensive way of acquiring signals generated by the brain. These signals are influenced by different types of brain stimuli such as eye blinks but they are also includes a large amount of noise, e.g., generated by the board. However, the noise can be removed with the help of proven filters. In this aspect, the intention of this work is to demonstrate how using different type of filters, it is possible to clean the noise from the brain signals acquired using an encephalography devices (such as Cytonbiosensing board) which are generated when a user blinks his/her eyes and classify them in different type of blinks. We have chosen the study of eye blink brain signals, since, they present a wide range of real-life applications. Our model includes a simple algorithm that classifies user-generated eye blinks into short intended blinks and long composed blinks. Experimental results of the proposed model show an accuracy of $96 \%$ which enables the development of real-life applications that do not require real-time control such as IoT devices.
\end{abstract}

Key words: OpenBCI, eye blink detection, digital signal processing, electroencephalography, EEG, feature extraction, eyelids, signal analysis

\section{INTRODUCTION}

Noninvasive Electroencephalography (EEG) devices such as the Muse Headband, Emotiv Epoc, NeuroSky TGAT1/TGAM1 and Cyton Biosensing board (OpenBCI V3) acquire electrical signals generated by the brain while the user is performing any type of actions such as blinking, meditating or even staring at a lights with different frequencies. However, those signals are raw data that include a considerable amount of noise produced by the amplifier of EEG devices or external factors. In this sense, in order to use the signals gathered by the EEG device in real-life applications, the use of different filters is needed to clean the data.

In this situation, this research looks for applying different filters models, i.e., high-pass, band-pass and band-stop filters to clean the signals gathered by the OpenBCI V3 when the user make voluntary blinks and classifying those signals into two different groups, i.e., short intended blinks and long composed blinks.

We have chosen the study of eye blink brain signals, since, they can be used in different real life applications. For example, Dhanush et al. (2018) have determined a person's response credibility based in eye-blinking count (Jamil et al., 2016) has proposed an automobile accident prevention using eye blinking detection, additionally (Rani and Mansor, 2009; Sourab et al., 2014; Zavala et al., 2018) have proposed home automation solutions using Internet of Thing (IoT) devices controlled by eye blinks and Hori et al. (2004) have proposed communicating devices for disabled individuals controlled by voluntary eye blinking.

Background: The offset produced by the DC amplifier is also known as Slow Cortical Potentials (SCP) an it is described by Birbaumer (1999). SCPs are positive or negative changes in an EEG that can last from $300 \mathrm{msec}$ to some seconds. Negative changes are a threshold regulation mechanism for local excitatory mobilization while positive changes show inhibition of cortical networks. This compensation generates Event-Related Potentials (ERP) that include Contingent Negative Variation CNV), Motion-Related Potentials (MRP), P300 and N400 Stern et al. (2001). The SCPs takes importance in this research, since, the oscillations in cortical networks are affected by events that disturb vision in specific frequency ranges (Freeman and van Dijk, 1987).

Since, Brain-Computer Interfaces (BCI) work with signals emitted by the brain, it's critical to not only acquire these signals (e.g., through nodes to read neuroelectric signals like EEG) but improve the quality of them by reducing noise and artifacts (Nam et al., 2018). This improvement can be achieved by processing the signal using a series of filters. In this situation, the signal processing in BCI has become a very important field in $\mathrm{BCI}$ researches and it has resulted in the

Corresponding Author: Sang Guun Yoo, Departamento de Informática y Ciencias de la Computación, Escuela Politécnica Nacional, Quito, Ecuador 
development of different types of tools. For example, SciPy is a Python-based open-source software for mathematics, science and engineering which has signal processing libraries with useful filtering functions.

Digital Signal Processing (DSP) refers to the process of analyzing and modifying a signal to optimize or improve its efficiency or performance (Meddins, 2000). Generally, a DSP system has an analog-to-digital converter (previously described), a digital processor that modifies the digital values from the previous module according to the required processing (e.g., amplification, rectification, filtering) and a digital-to-analog converter that output the reconstituted analog signal.

Filtering is one the main applications of DSP in the BCI research field. A filter can be classified into 4 types: high-pass, low-pass, band-stop or band-pass. The high-pass filter is a filter designed by starting with a low-pass filter and then converting it into the desired response (Christiano and Fitzgerald, 2003). It is commonly used to remove low-frequency offsets from a signal. A band-stop filter is a spectral filter that attenuates or rejects frequencies between two cut-off frequencies. A band-pass filter allows the transition of a certain range of frequencies of a signal, filtering low and high frequencies at the same time and attenuating the rest. In simpler terms, it's the application of a high-pass and a low-pass filter (Christiano and Fitzgerald, 2003). And all those digital filters can be implemented as an Infinite Impulse Response (IIR) or a Finite Impulse Response (FIR) system (Meddins, 2000). The main difference is that FIR filters just apply the mathematical definition of the filter with all the input values while IIR filters use previous outputs as feedback for the system too. This small difference makes IIR have a recursive behavior, so, a simple impulse can emit values infinitely, making it faster, computational simpler but unstable.

All the digital filters mentioned above are implemented in SciPy through the Butterworth digital filter which needs the order of the filter and the values of the cut-off frequencies. In order to be implemented with SciPy, the cut-off frequencies must be relative to the Nyquist frequency which is achieved through the following formula:

$$
\mathrm{Wn}=\frac{\text { cutoff } \_ \text {Hz }}{\text { nyq_fs_Hz }}
$$

Where:

Wn : The digital cutoff frequency

cutoff_Hz : The cut-off frequency

nyq_fs_Hz : The Nyquist frequency which is half the sampling frequency

\section{MATERIALS AND METHODS}

Implementation: The purpose of this research to present a way to detect user-generated blinks using the Cyton Biosensing board (OpenBCI V3). This board is composed of several important pieces that together make possible access to human brain waves. One of those components is the Texas Instruments ADS1299 24-bit electroencephalography which has an eight-channel delta-sigma analog-to-digital converter (ADS1299, 2017). This chip is used in the OpenBCI V3 to acquire and digitize biopotential signals OpenBCI-Open Source Biosensing Tools (Anonymous, 2019) and has other capabilities such as the generation of internal signals for testing and calibration and lead-off detection for ensuring that the electrodes are in contact with the subject.

The ADS1299 chip has a built-in amplifier used to increase signals. In other words, this chip is a Direct-Coupled amplifier (DC Amplifier). In this type of amplifier, the output of the first stage is connected directly as the input of the next stage without any coupling device (Kumar and Jain, 2007) allowing the amplification of signals with very low frequency. However, it presents a significant offset that can be positive or negative over time. This situation can cause problems in the process of analyzing the acquired signals and the problem includes the process of detecting eyeblinks. Luckily the use of proven filters can help clean the signals. A high pass filter can help remove the offsets caused by the DC amplifier while band-pass and band-stop or band-rejection filters are usually used to remove noises and power-line data from gathered signals (Nam et al., 2018). The order in which these filters are applied does not matter.

Taking this into account, we have proposed the model shown in Fig. 1 to detect user generated blinks which will be classified later into short intended blinks and long composed blinks. We have chosen these two types of blinking taking into consideration that the EEG device will be worn by the user constantly and the system must not consider the involuntary blinks performed by the user in his/her normal activities.

The raw signals for the experiments of the proposed model were acquired using the Cyton Biosensing board (OpenBCI V3) with one active reusable flat snap electrode (TDE-202) located on the surface of the scalp (FP1 or FP2), according to the extended 10-20 system indicated by Klem et al. (1999) and the sampling frequency was set to $250 \mathrm{~Hz}$ considering that the ADS 1299 presents a small offset over time (we have removed this using a high-pass filter at $0.5 \mathrm{~Hz}$ ). In order to remove the highest noise inside the signals, two band-stop filters 


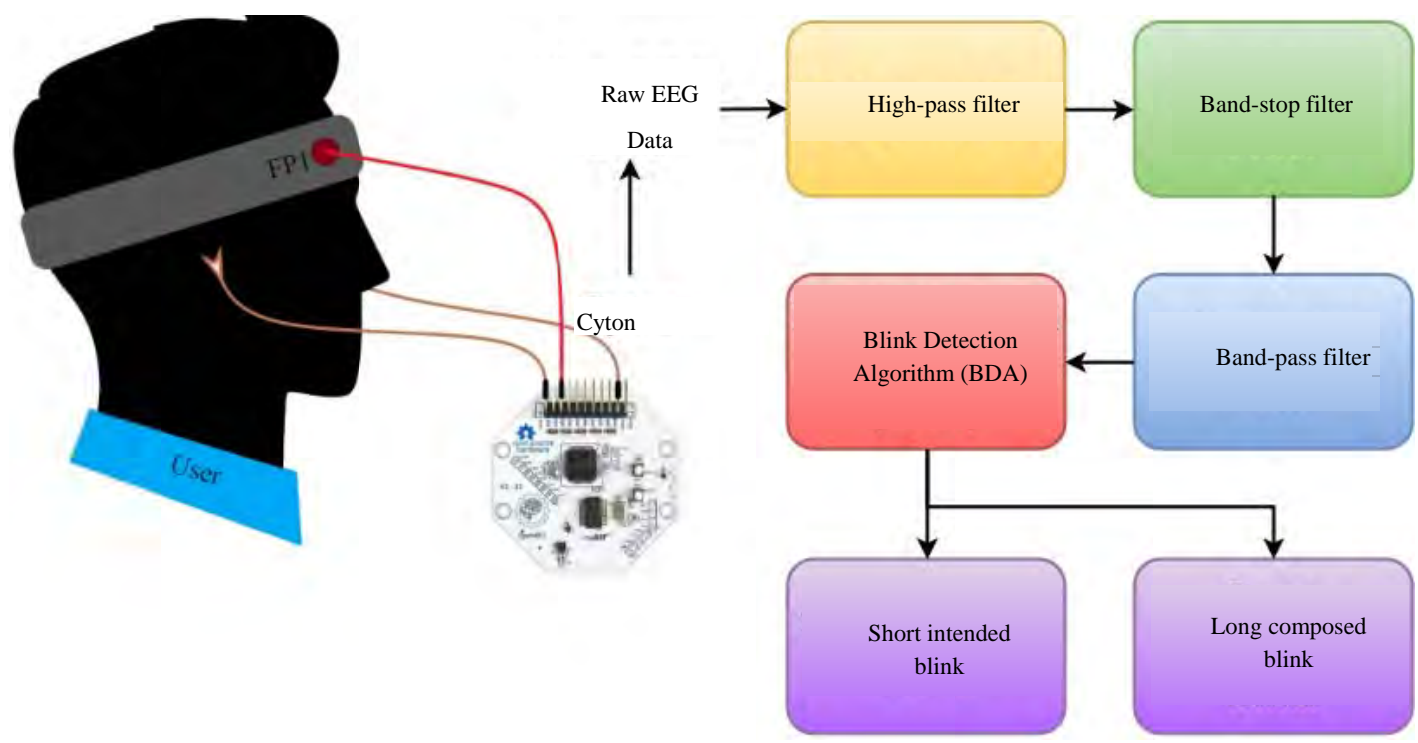

Fig. 1: Complete diagram of the system
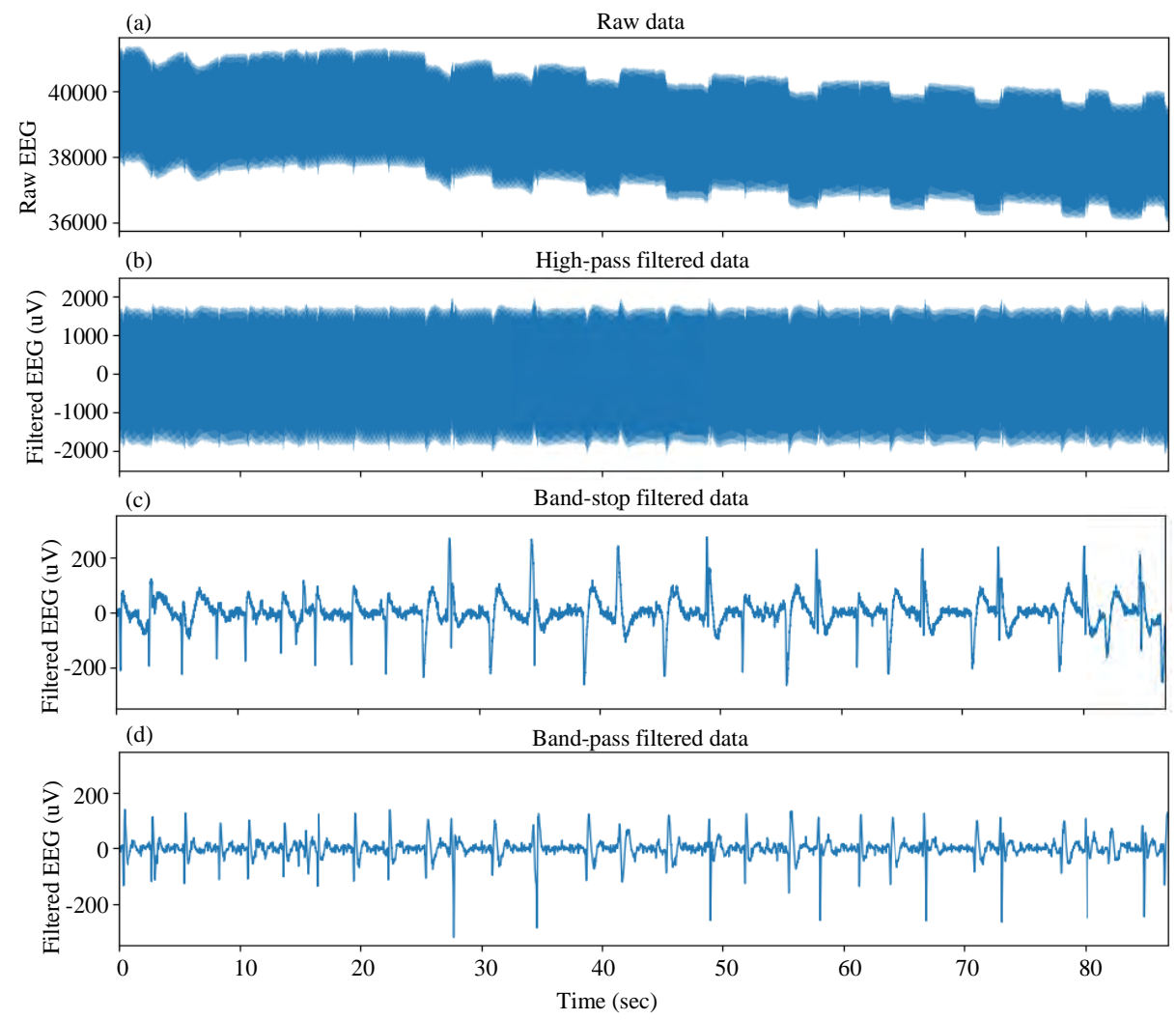

Fig. 2(a-d): Signal transition between each filter

where used. The first stop-band frequencies were set at 57-63 $\mathrm{Hz}$ and the second one was set at $117-123 \mathrm{~Hz}$. Then, a band-pass filter was used between 1 and $25 \mathrm{~Hz}$ to generate a more accurate signal and remove unwanted noise and artifacts. Figure 2 shows how the signal changes after each filter. 


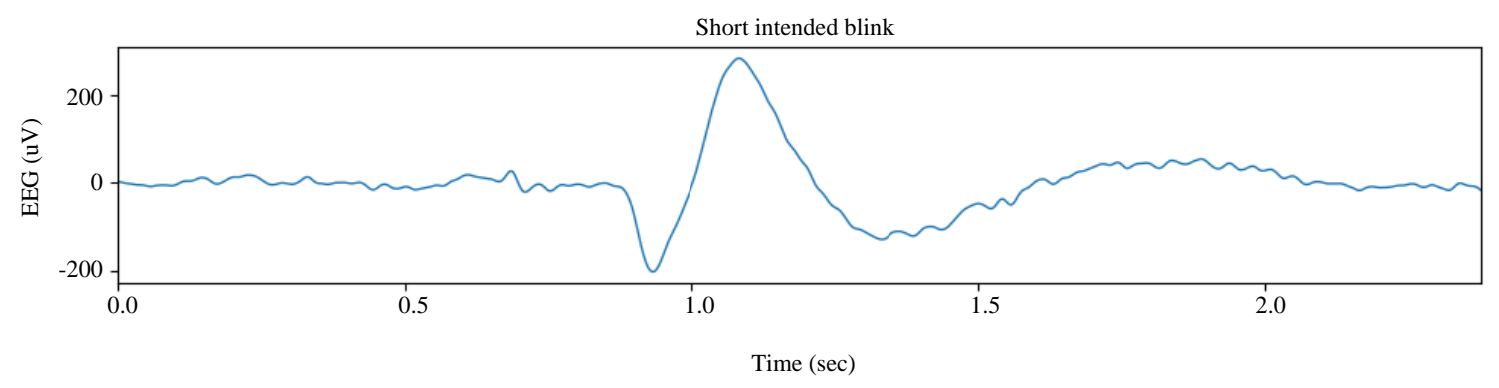

Fig. 3: Short intended blink

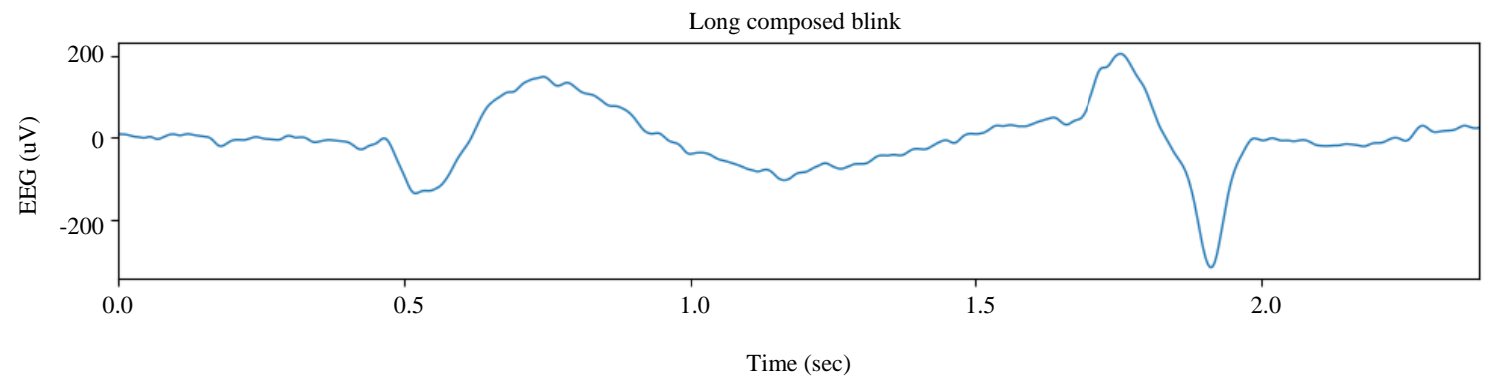

Fig. 4: Long composed blink

Once the signals are processed using the aforementioned filters, they enter to the proposed Blink Detection Algorithm (BDA). In this algorithm, the signals are classified into short intended blinks and long composed blinks. A short-intended blink is detected when the signal drops below $-125 \pm 15 \mathrm{uV}$, then rises above $125 \pm 15 \mathrm{uV}$ and then goes in a state of normality (between -45 and $45 \mathrm{uV}$ ) in a window of $2.5 \mathrm{sec}$ (as shown in Fig. 3). A long composed blink is detected when the user closes their eyes for $2 \pm 0.3 \mathrm{sec}$, opens them and then blinks quickly again; all of these steps are executedina time frame of $2.5 \mathrm{sec}$. When this happens, the signals drops below $-125 \pm 15 \mathrm{uV}$, rises above $125 \pm 15 \mathrm{uV}$, enters a state of normality, then rises above $125 \pm 15 \mathrm{uV}$ and then drops below $-125 \pm 15 \mathrm{uV}$ again; similar to short intended blinks, all the steps are done in a window of $2.5 \mathrm{sec}$ (Fig. 4).

\section{RESULTS AND DISCUSSION}

Experimentation: To verify the effectivity of blink detection, seventeen 21-24 years old healthy participants performed two tasks. First, each experiment subject performed a total of 40 short intended blinks and then, they performed 40 long composed blinks. In total, data of 1360 blinks were acquired. After the treatment of the raw data, among the 1360 blinks, only 35 short blinks and 49 long composed blinks were misclassified. This tells us that the model showed a $97.426 \%$ success rate in classifying short intended blinks and $96.397 \%$ in classifying long composed blinks.

\section{CONCLUSION}

Through the performed research and experimentation, it was possible to detect two different types of blinks (short intended blinks and long composed blinks) made by a person. With a performance of 97.426 and $96.397 \%$ for classifying short intended blinks and long composed blinks, respectively. We believe that these values are acceptable considering that the process is done in real-time. Each of the filters maintains its own relevance when processing the data, this implies that if a filter is deleted, the desired results will not be obtained. One limitation of the present model is that the user has a $2.5 \mathrm{sec}$ window to complete the short intended blinks and long composed blinks which means that they must wait $2.5 \mathrm{sec}$ between each blink. However, applications that do not require high-speed control could be developed using the proposed eye blink detection model (e.g., the control of IoT devices such as televisions or light bulbs).

\section{ACKNOWLEDGEMENT}

The researchers gratefully acknowledge the financial support provided by the Escuela Politécnica Nacional, for the development of the project PIS-17-15-“Control de Dispositivos a través del Pensamiento (Ondas Cerebrales).

\section{REFERENCES}

ADS1299, 2017. Low-noise, 8-channel, 24-bit analog-to-digital converter for biopotential measurements. Texas Instruments, Dallas, Texas, USA. http://www.ti.com/product/ADS1299 
Anonymous, 2019. Open source biosensing tools (EEG, EMG, EKG and more). OpenBCI, Dallas, Texas, USA. https://openbci.com/

Birbaumer, N., 1999. Slow cortical potentials: Plasticity, operant control and behavioral effects. Neuroscientist, 5: 74-78.

Christiano, L.J. and T.J. Fitzgerald, 2003. The band pass filter. Int. Econ. Rev., 44: 435-465.

Dhanush, T., T.S. Sharmila and J.S. Jennifer, 2018. Determining Response credibility by blink count. Proceedings of the 2018 International Conference on Recent Trends in Advance Computing (ICRTAC'18), September 10-11, 2018, IEEE, Chennai, India, pp: 143-148.

Freeman, W.J. and B.W. van Dijk, 1987. Spatial patterns of visual cortical fast EEG during conditioned reflex in a rhesus monkey. Brain Res., 422: 267-276.

Hori, J., K. Sakano and Y. Saitoh, 2004. Development of communication supporting device controlled by eye movements and voluntary eye blink. Proceedings of the 26th Annual International Conference on Engineering in Medicine and Biology Society, September 1-5, 2004, IEEE, San Francisco, California, USA., pp: 4302-4305.

Jamil, T., I. Mohammed and M.H. Awadalla, 2016. Design and implementation of an eye blinking detector system for automobile accident prevention. Proceedings of the 2016 IEEE SoutheastCon, March 30-April 3, 2016, IEEE, Norfolk, Virginia, USA., pp: $1-3$.

Klem, G.H., H.O. Luders, H.H. Jasper and C. Elger, 1999. The ten- twenty electrode system of the International Federation: The International Federation of clinical neurophysiology. Electroencephalogr Clin Neurophysiol, 52: 3-6.
Kumar, B. and S.B. Jain, 2007. Electronic Devices and Circuits. 1st Edn., PHI Learning Pvt. Ltd., New Delhi, India, ISBN: 978-81- 203-2981-2, Pages: 724.

Meddins, R., 2000. Introduction to Digital Signal Processing. Newnes, Oxford, England, ISBN: 9780080535005, Pages: 161.

Nam, C.S., A. Nijholt and F. Lotte, 2018. Brain-Computer Interfaces Handbook: Technological and Theoretical Advances. CRC Press, Boca Raton, Florida, USA., ISBN: 9781351231930 , Pages: 788.

Rani, M.S.B.A. and W.B. Mansor, 2009. Detection of eye blinks from EEG signals for home lighting system activation. Proceedings of the 2009 6th International Symposium on Mechatronics and its Applications, March 23-26, 2009, IEEE, Sharjah, UAE., pp: 1-4.

Sourab, B.S., R.C. HS and S. D'Souza, 2014. Implementation of home automation using eye blink sensor. Proceedings of the 2014 International Conference on Electronics, Communication and Computational Engineering (ICECCE'14), November 17-18, 2014, IEEE, Hosur, India, pp: 242-244.

Stern, R.M., W.J. Ray and S.R. Morris, 2001. Psychophysiological Recording. 2nd Edn., Oxford University Press, Oxford, UK., ISBN: 9780195113594, Pages: 282.

Zavala, S.P., J.L.L. Bayas, A. Ulloa, J. Sulca, J.L.M. Lopez and S.G. Yoo, 2018. Brain computer interface application for people with movement disabilities. Proceedings of the International Conference on Human Centered Computing (HCC’18), December 5-7, 2018, Springer, Cham, Switzerland, pp: 35-47. 